\title{
Novel hybrid biofilm system using synthetically engineered curli fibres
}

\section{Nouveau système de biofilm hybride utilisant des fibres de curli synthétiques}

Harshini Ramesh, Keerthana Pasumarthi, Maggie Hou and Jennifer Lee

\begin{abstract}
Hydraulic fracturing, a popular mining technique, generates heavy metal contamination in nearby freshwater aquifers. This poses a threat to both the surrounding ecosystems and human health if exposed. Existing methods of heavy metal removal can produce additional hazardous byproducts. This proposal presents the use of a hybrid biofilm filter containing graphene and curli fibres with metal binding sites. Curli fibres are amyloid fibrils found on the extracellular biofilm of Escherichia coli (E coli.). Through the use of plasmid vectors, E. coli will be engineered to produce secreted curli fibres with metal-binding residues. The stability and cohesive properties of the curli fibres augments the adherence to the graphene scaffolding, thus allowing for generation of a hybrid biofilm. With the filtration design and various experimental controls proposed, this model is ready for empirical proof of concept and subsequent quantitative optimization.
\end{abstract}

\section{Résumé}

La fracturation hydraulique, technique d'extraction populaire, génère une contamination par les métaux lourds dans les aquifères d'eau douce situés à proximité. Cela constitue une menace à la fois pour les écosystèmes environnants et pour la santé humaine. Les méthodes actuelles d'élimination des métaux lourds peuvent produire des sous-produits dangereux supplémentaires. Cette proposition présente l'utilisation d'un filtre à biofilm hybride contenant du graphène et des fibres de curli avec des sites de liaisons métalliques. Les fibres de curli sont des fibrilles amyloïdes retrouvées sur le biofilm extracellulaire d'Escherichia coli (E coli.). Grâce à l'utilisation de vecteurs plasmidiques, l'E. coli sera manipulé pour produire des fibres de curli sécrétées avec des résidus de liaison aux métaux. La stabilité et les propriétés cohésives des fibres de curli augmentent l'adhérence à l'échafaudage de graphène, permettant ainsi la génération d'un biofilm hybride. Avec la conception de la filtration et les différents contrôles expérimentaux proposés, ce modèle est prêt pour la validation empirique du concept et l'optimisation quantitative subséquente.

\footnotetext{
*McMaster University, Hamilton, Ontario, Canada

Université McMaster, Hamilton, Ontario, Canada

${ }^{\dagger}$ Lower division / Division inférieure

$\ddagger$ 2nd place / 2ème place
} 